\title{
Structural and Electronic Flexibility in Hydrides of Zintl Phases with Tetrel-Hydrogen and Tetrel-Tetrel Bonds
}

\author{
Henry Auer, ${ }^{[a]}$ Robert Schlegel,${ }^{[b]}$ Oliver Oeckler, ${ }^{[b]}$ and Holger Kohlmann ${ }^{*[a]}$
}

\begin{abstract}
The hydrogenation of Zintl phases enables the formation of new structural entities with main group elementhydrogen bonds in the solid state. The hydrogenation of $\mathrm{SrSi}$, $\mathrm{BaSi}$ and $\mathrm{BaGe}$ yields the hydrides $\mathrm{SrSiH}_{5 / 3-x}, \mathrm{BaSiH}_{5 / 3-x}$ and $\mathrm{BaGeH}_{5 / 3-x}$. The crystal structures show a six-fold superstructure compared to the parent Zintl phase and were solved by a combination of X-ray, neutron and electron diffraction and the aid of DFT calculations. Layers of connected $\mathrm{HSr}_{4}\left(\mathrm{HBa}_{4}\right)$ tetrahedra containing hydride ions alternate with layers of infinite single- and double-chain polyanions, where hydrogen atoms are covalently bound to silicon and germanium. The idealized formulae $A e T t \mathrm{H}_{5 / 3}$ ( $A e=$ alkaline earth, $T t=$ tetrel $)$ can be rationalized with the Zintl-Klemm concept according to $\left(A e^{2+}\right)_{3}\left(T t \mathrm{H}^{-}\right)\left(T t_{2} \mathrm{H}^{2-}\right)\left(\mathrm{H}^{-}\right)_{3}$, where all Tt atoms are three-binding. The non-stoichiometry $\left(\mathrm{SrSiH}_{5 / 3-x}, \quad x=0.17(2) ; \quad \mathrm{BaGeH}_{5 / 3-x}\right.$, $\mathrm{x}=0.10(3)$ ) can be explained by additional $\pi$-bonding of the $\mathrm{Tt}$ chains.
\end{abstract}

The formation of covalent element-hydrogen bonds is a common theme in synthetic chemistry. Recently, new polymeric triel- and tetrel-hydrogen units were found in the solid state by hydrogenation of Zintl phases or by direct synthesis in hydrogen atmosphere.$^{[1]}$ Examples are triel based polyanions ${ }_{\infty}^{2}\left[\mathrm{TrH}^{\mathrm{H}}\right]$ and $\infty^{2}\left[(T r H)^{-} T t\right]$ forming puckered honeycomb-layers with hydrogen bound to the triel atom in $A e T_{2} \mathrm{H}_{2}$ and $A e \operatorname{TrTtH}(A e=\mathrm{Sr}$, Ba; $\operatorname{Tr}$ $=\mathrm{Al}, \mathrm{Ga} ; \mathrm{Tt}=\mathrm{Si}, \mathrm{Ge}, \mathrm{Sn})$, respectively, ${ }^{[1]}$ and pseudo-alkane like moieties, e. g. neopentane like $\left[\mathrm{Ga}\left(\mathrm{Ga}_{3} \mathrm{H}_{5}\right)_{4}\right]^{5-{ }^{[2]}}$ Hydrogen containing tetrel based anions comprise monomeric $\mathrm{SiH}_{3}{ }^{-}$in $\mathrm{ASiH}_{3}(A=\mathrm{K}-\mathrm{Cs}),{ }^{[3,4]}$ infinite single chains ${ }_{\infty}^{1}\left[\mathrm{SiH}^{-}\right]$in $\mathrm{BaSiH}_{2-x},{ }^{[5]}$ and infinite triple chains ${ }_{\infty}^{1}\left[\mathrm{HTt}_{6} \mathrm{H}^{6-}\right]$ in $\mathrm{CaSiH}_{4 / 3-x}, \mathrm{SrGeH}_{4 / 3-x}$ and $\mathrm{BaSnH}_{4 / 3-x^{[5,6]}}$ For the latter, the parent Zintl phases AeTt crystallize in the CrB structure type (space group $\mathrm{Cmcm}$ ) and exhibit infinite polyanionic silicon and germanium zigzag chains $\infty^{1}\left[T t^{2}\right]$ in agreement with the Zintl-Klemm concept. Since the hydrogenation of $\mathrm{CaSi}$ to $\mathrm{CaSiH}_{4 / 3-x}$ is reversible, this system is a potential hydrogen storage material. ${ }^{[6]}$ The hydrogenation of Zintl

[a] Prof. Dr. Holger Kohlmann, Henry Auer Institute of Inorganic Chemistry

Leipzig University

Johannisallee 29

04103 Leipzig

Germany

holger.kohlmann@uni-leipzig.de

[b] Prof. Dr. Oliver Oeckler, Robert Schlegel

Institute of Mineralogy, Crystallography and Materials Science Leipzig University

Scharnhorststraße 20

04275 Leipzig

Germany

Supporting information for this article is given via a link at the end of the document. phases is a very promising yet barely explored synthetic pathway to new main group element-hydrogen entities in the solid state. In this contribution, we extend this approach and report on new main group-hydrogen structural units in the hydrides of SrSi, BaSi and $\mathrm{BaGe}$. We will focus on the rationale behind the multitude of polyanionic structural motifs, the formation of new tetrel-tetrel and tetrel-hydrogen bonds in the solid and the hydrogen content of the hydrides.

The Zintl phases SrSi and BaGe were hydrogenated at $453 \mathrm{~K}$ and $473 \mathrm{~K}$ under hydrogen gas pressures of 9.0 and $5.0 \mathrm{MPa}$, respectively, forming $\mathrm{SrSiH}_{5 / 3-\mathrm{x}}, \mathrm{x}=0.17(2)$ (1.27 wt-\% hydrogen, elemental analysis: $1.16(6)$ wt- $\%)$, and $\mathrm{BaGeH}_{5 / 3-\mathrm{x}}$, $x=0.10(3)(0.74$ wt- $\%$ hydrogen, elemental analysis: $0.73(3)$ wt$\%$, see Supplementary Information for details). The hydrides are gray powders sensitive against air and moisture. Exposed to air, they yield amorphous products. The color of $\mathrm{BaGeH}_{5 / 3-x}$ changes to yellow in the course of this reaction, which is probably caused by hydrolysis to polygermene, $\left(\mathrm{GeH}_{2}\right)_{x \cdot{ }^{[7]}} \mathrm{A}$ similar color change change was seen for the moisture induced decomposition of $\mathrm{SrAIGeH}$ and BaAIGeH where the formation of $\mathrm{Ge}-\mathrm{H}$ bonds was followed by IR spectroscopy. ${ }^{[8]}$
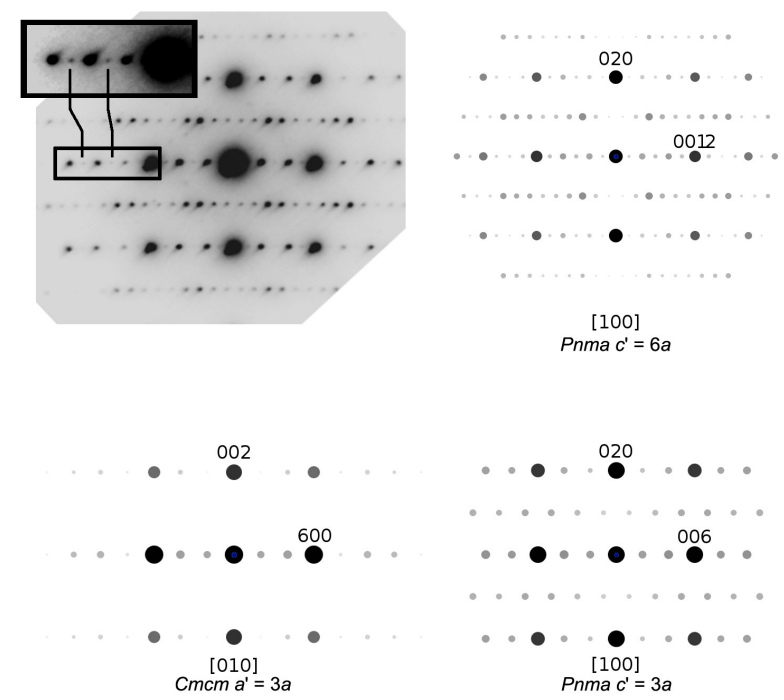

Figure 1. Top left: SAED pattern of a $\mathrm{SrSiD}_{5 / 3-x}$ crystal (inset does not scale linearly). Top right: Simulation using the final model (Pnma, sixfold superstructure regarding the parent Zintl phase), all observed reflections are explained. Bottom left: Simulation using the model with half-occupied hydrogen sites $(\mathrm{Cmcm}$, threefold superstructure), rows of reflections are missing due to $C$ centering. Bottom right: Simulation based on the $\mathrm{CaSiH}_{1.3}$ structure type, additional row of reflections explained, but mismatch for position and quantity. For corresponding stereographic projection and further SAED patterns with simulations see supplementary information (Figs. S4, S5). 
The crystal structures of $\mathrm{SrSiH}_{5 / 3-x}, \quad x=0.17(2)$ and $\mathrm{BaGeH}_{5 / 3-x}, x=0.10$ (3) were elucidated by a combination of electron, X-ray and neutron powder diffraction. The latter two clearly showed a threefold superstructure regarding the parent Zintl phase with $a_{\text {hydride }} \approx 3 a_{\text {zintl phase }}$, i. e. perpendicular to the $\infty^{1}\left[T t^{2}\right]$ zigzag chains. Structure solution and refinement first led to a model in space group type $\mathrm{Cmcm}$ with two fully and two ca. $50 \%$ occupied hydrogen sites. Selected-area electron diffraction (SAED) patterns of the $\mathrm{Sr}$ compound indicated a further cell doubling, i.e. a six-fold superstructure (Fig. 1). With the help of DFT relaxations of various crystal structure candidates, a model with completely ordered hydrogen distribution was found in space group Pnma ( $c_{\text {hydride }} \approx 6 a_{\text {zintl phase }}$, Fig. 2). Joint Rietveld refinements using laboratory $\mathrm{X}$-ray and neutron diffraction data of the deuterides revealed vacancies of up to ca. $20 \%$ on some of the hydrogen sites (for details of the structure solution and refinement cf. Supplementary Information). SAED patterns of a BaSi hydride sample suggested the presence of a phase isotypic to $\mathrm{SrSiH}_{5 / 3-x}$ and $\mathrm{BaGeH}_{5 / 3-x}$. The formerly unassigned side phase in $\mathrm{BaSiH}_{2-\mathrm{x}}$ samples ${ }^{[5]}$ is thus identified as $\mathrm{BaSiH}_{5 / 3-x}$ (Figs. S8S10 in Supplementary Information).
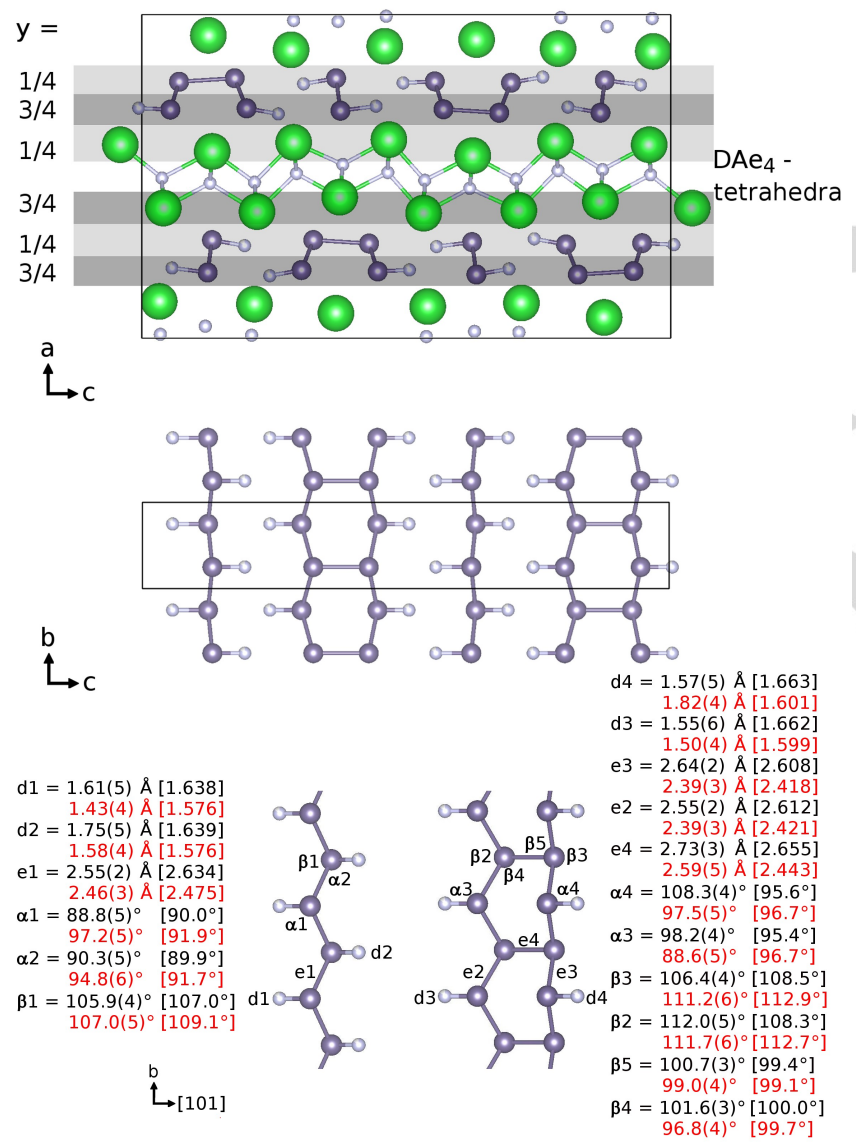

Figure 2. Projections of the crystal structure of $\mathrm{BaGeD}_{5 / 3-x}$ (top) and polyanionic partial structure (middle, bottom), large spheres (green): $\mathrm{Ba}$ medium spheres (gray): Ge, small spheres (white): H (D). Experimental interatomic distances and angles in comparison to corresponding ones calculated by DFT [in brackets] (first line (black): $\mathrm{BaGeD}_{5 / 3-x}$, second line (red): $\mathrm{SrSiD}_{5 / 3-\mathrm{x}}$ ).
The hydrides of SrSi and BaGe are non-stoichiometric with respect to hydrogen and crystallize in space group type Pnma $\left(\mathrm{SrSiH}_{5 / 3-\mathrm{x}}\right.$, hydride: $a=15.1009(6) \AA, b=3.95121(18) \AA, c=$ 24.6298(12) $\AA$, deuteride: $a=15.070(2) \AA, b=3.9503(2) \AA, c=$ 24.642(2) $\AA$; $\mathrm{BaGeH}_{5 / 3-\mathrm{x}}$, hydride: $a=15.8253(9) \AA, \quad b=$ 4.2308(2) $\AA, c=25.8728(14) \AA$; deuteride: $a=15.804(3) \AA, b=$ 4.2293(6) $\AA, c=25.855(4) \AA{ }^{[9]}$ Tab. S5, S7 and S8 in Supplementary Information). The crystal structures can be rationalized in their idealized composition $A e T t \mathrm{H}_{5 / 3}$ according to the Zintl concept as $\left(A e^{2+}\right)_{3}\left(T t \mathrm{H}^{-}\right)\left(T t_{2} \mathrm{H}^{2-}\right)\left(\mathrm{H}^{-}\right)_{3}$, i. e. they contain hydride anions as well as hydrogen covalently bound to the polyanions. Having a formal charge of 1-, all tetrel atoms are expected to form three covalent bonds, either to three tetrel atoms or to two tetrel and one hydrogen atom. The polyanions show two structural motifs (Fig. 2). In the first one, the parallel $\infty^{1}\left[T^{2}\right]$ zigzag chains, which are already present in the parent Zintl phase, are inclined towards each other and form additional tetrel-tetrel bonds. This results in double chains ${ }_{\infty}^{1}\left[T_{2} \mathrm{H}^{2-}\right]$ $\left(=_{\infty}{ }^{1}\left[\mathrm{HTt}-\mathrm{Tt}_{2}-\mathrm{TtH}^{4}\right]\right.$, Fig. 2), where every other tetrel atom covalently binds to a hydrogen atom. This polyanion may also be described as a chain of condensed six-membered rings of tetrel atoms in boat conformation. The second motif is a zigzag chain $\infty_{\infty}^{1}\left[\mathrm{TtH}^{-}\right]$as obtained for $\mathrm{BaSiH}_{2-x,}{ }^{[5]}$ running parallel to the double chain. The crystal structure is clearly stabilized by strong ionic bonding from additional hydride ions in $\mathrm{Ae}_{4}$ tetrahedra (Fig. 2). Crystal structure (for both) and hydrogen content (for the BaSi hydrides) contradict an earlier report of hydrides of AeSi Zintl phases, where, however, hydrogen positions were not determined. ${ }^{[10]}$ The crystal structure shows a close relationship to the parent Zintl phase. The chain direction of the polyanions ([001] in the Zintl phase, [010] in the hydride) shrinks by $2 \%$ upon formation of the hydride. A considerable expansion of more than $30 \%$ occurs in the direction perpendicular to the $\mathrm{HAe}_{4}$ tetrahedra sheets ([010] in the Zintl phase, [100] in the hydride) (Fig. 2) due to the incorporation of hydrogen. Connecting the polyanions of the Zintl phase to double chains leads to a reduction of $15 \%$ in that direction ([100] in the Zintl phase, [001] in the hydride, accounting for the sixfold superstructure). These strongly anisotropic effects of hydrogenation certainly reflect the bonding situation, which was investigated by quantummechanical calculations (DFT).

In zigzag chain direction [010], tetrel-tetrel bonds are $2.41 \AA$ [2.438 $\AA$ ] (Si) and $2.58 \AA$ [2.633 $\AA$ ] (Ge) (DFT results in brackets, Fig. 2, e1-3) while the additional chain-connecting bond (Fig. 2, e4) is longer with $2.59 \AA(\mathrm{Si})$ and $2.73 \AA(\mathrm{Ge})$. This is not the case for DFT calculated values of the hypothetical stoichiometric composition $A e T t \mathrm{H}_{5 / 3}(2.443 \AA(\mathrm{Si})$ and $2.655 \AA(\mathrm{Ge}))$. The tetreltetrel distances are comparable to those in the pristine Zintl phases. Si-Si-Si angles range from $96^{\circ}$ to $111^{\circ}$ (Ge-Ge-Ge: $101^{\circ}$ to $\left.107^{\circ}\right)$ and are in a similar range as in the chair configuration of black phosphorus (P-P-P: $102.09(8)^{\circ}$ and $\left.96.34(11)^{\circ}\right)^{[11]}$ and gray arsenic (As-As-As: $\left.96.64(6)^{\circ}\right),{ }^{[12]}$ respectively.

Refined distances Si-D / Ge-D (averaged $d(G e-D)=1.62 \AA$ $[1.650 \AA]$, averaged $d(S i-D)=1.58 \AA[1.588 \AA]$; DFT results in brackets) indicate covalent interactions. Si-D distances are in between those found in $\beta-\mathrm{KSiD}_{3}(1.537(8)-1.545(6) \AA){ }^{[4]}$ and $\mathrm{BaSiD}_{2-x}(1.641(5) \AA),{ }^{[5]}$ while Ge-D distances are somewhat longer than in molecular $\mathrm{GeD}_{4}(1.517(3) \AA \text { at } 5 \mathrm{~K})^{[13]}$ or $\mathrm{SrGeD}_{4 / 3-x}$ $(1.521(9) \AA) .{ }^{[5]} \mathrm{Sr}-\mathrm{D}$ and Ba-D distances reach from 2.35(4) to $2.57(4) \AA$ and $2.41(6)$ to $2.73(6) \AA$, respectively, and are comparable to those in $\mathrm{DAe} \mathrm{e}_{4}$ tetrahedra of the binary deuterides $\mathrm{SrD}_{2}$ and $\mathrm{BaD}_{2}$ (average $2.44 \AA$ and $2.62 \AA$, respectively), ${ }^{[14]}$ thus 
underlining the ionic nature of bonding within the $\mathrm{HAe}$ tetrahedra sheets.

Hydrogen atoms covalently bound to the polyanions do not show full occupation (85(2)\% in $\mathrm{BaGeH}_{5 / 3-x}, 77.2(8) \%$ in $\left.\mathrm{SrSiH}_{5 / 3-x}\right)$. There is no hint to further ordering, not even at low temperature, as studied by neutron diffraction on $\mathrm{BaGeH}_{5 / 3-x}$ at $10 \mathrm{~K}$ (Supplementary information, S3). Such hydrogen-atom vacancies are an intrinsic feature of Zintl-phase hydrides $A e T t \mathrm{H}_{y}$ $(A e=\mathrm{Ca}-\mathrm{Ba}, \mathrm{Tt}=\mathrm{Si}-\mathrm{Sn}, 1<\mathrm{y}<2){ }^{[5]}$ They may be related to additional $\pi$-bonding due to depopulation of $\pi^{*}$ bands upon the formation of hydride anions. The effect of $\pi$-bonding has been discussed in detail for the parent Zintl-phase silicides. ${ }^{[15-17]}$ Additional $\pi$-bonding in non-stoichiometric samples can be assumed comparing DFT-derived bond lengths, which were calculated on idealized structures with full occupation. Experimentally observed tetrel-tetrel distances along the chain direction [010] are systematically shorter ( $\pi$-bonded) while chain-connecting bonds are longer than calculated distances.

The density of states (DOS) (Fig. 3) was calculated for $\mathrm{SrSiH}_{5 / 3}$ and $\mathrm{BaGeH}_{5 / 3}$ using the relaxed structures with an idealized full hydrogen site occupation. While the DOS of $\mathrm{BaGeH}_{5 / 3}$ hints at a band gap as expected for an electronprecise Zintl-phase hydride, $\mathrm{SrSiH}_{5 / 3}$ has significant electron density at the Fermi level and shows only a pseudo-gap. This could explain the non-stoichiometry, which is more pronounced in the latter compound, by electronic effects.

$\mathrm{BaGeH}_{5 / 3-x}$ and $\mathrm{SrSiH}_{5 / 3-x}$ may be considered the missing link between $\mathrm{SrGeH}_{4 / 3-x}$ with three tetrel zigzag chains connected to form a triple-chain polyanion ${ }_{\infty}^{1}\left[\mathrm{HGe}_{6} \mathrm{H}^{6}\right]$ and $\mathrm{BaSiH}_{2-x}$ with a single chain ${ }_{\infty}^{1}\left[\mathrm{SiH}^{-}\right]$. They underline the rich structural chemistry of the hydrides of Zintl phases and represent new structural motifs of main-group element chemistry. Examining the hydrides of CrB type Zintl phases AeTt $(A e=\mathrm{Ca}, \mathrm{Sr}, \mathrm{Ba} ; \mathrm{Tt}=\mathrm{Si}, \mathrm{Ge}, \mathrm{Sn})$ reveals a remarkable compositional, structural and electronic flexibility. There are two main positions for hydrogen atoms, in $\mathrm{Ae}_{4}$ tetrahedra and attached to tetrel polyanions, both of which may be under-occupied, and there are various types of polyanions in the solid state. Single tetrel zigzag chains may be saturated by hydrogen atoms like in $\mathrm{BaSiH}_{2-x},{ }^{[5]}$ or such chains may be connected to form double chains like in $A e T t_{5 / 3-x}$ or triple chains like in $\mathrm{CaSiH}_{1.3}{ }^{[6]} \mathrm{y}-\mathrm{SrGeH}_{4 / 3-x}$ and $\mathrm{BaSnH}_{4 / 3-\mathrm{x} x}{ }^{[5]}$ Alternatively, none of the above may occur for hydrogen-poor phases $\alpha-$ and $\beta-\mathrm{SrGeH}_{<1},{ }^{[18]}$ where the electronic balance is adjusted by the Ge-Ge bond length and the degree of $\pi$ bonding.

Thus, three types of structural and electronic compensation to the presence of hydrogen may be distinguished: (1) Increasing connectivity from two- to three-bonding by linking of single to double or triple chains, thus considering hydrogenation as an oxidation, e. g. $\left[\mathrm{Si}(2 \mathrm{~b})^{2}\right]_{x}+1 / 2 \mathrm{H}_{2}=\left[\mathrm{Si}(3 \mathrm{~b})^{-}\right]_{x}+\mathrm{H}^{-;}$(2) covalent bonds to hydrogen, e. g. $\left[\mathrm{Si}^{2-}\right]_{x}+\mathrm{H}_{2}=\left[\mathrm{SiH}^{-}\right]_{x}+\mathrm{H}^{-}$; (3) increasing $\pi$-bonding in the polyanionic chains by depopulating $\pi^{*}$ bands upon formation of hydride anions. The balance between (1) and (2) strongly depends on geometrical restrictions implied by the alkaline earth atoms. With their increasing size, binding hydrogen covalently (2) outweighs the linking of polyanions (1), because of its higher space requirements. The highest degree of condensation is found in $\mathrm{CaSiH}_{1.3},{ }^{[6]} \mathrm{Y}$ $\mathrm{SrGeH}_{4 / 3-x}$ and $\mathrm{BaSnH}_{4 / 3-x,{ }^{[5]}}$ where the layers of hydride-filled $\mathrm{Ae}_{4}$ tetrahedra are strongly puckered to fit to the translational period of the polyanionic layers. Complete condensation to

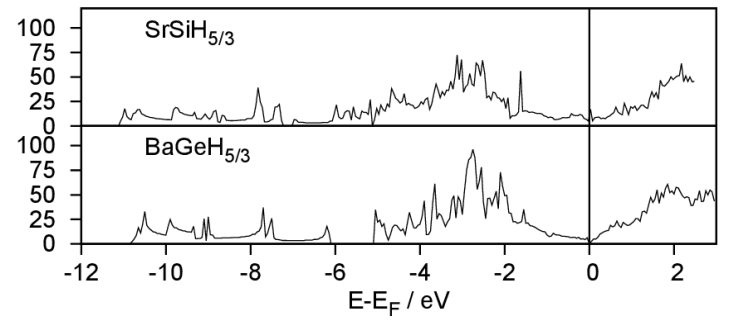

Figure 3. Electronic density of states of $\mathrm{SrSiH}_{5 / 3}$ (top) and $\mathrm{BaGeH}_{5 / 3}$ (bottom)

hydrogen-free layer-like polyanions $\infty_{\infty}^{2}[T t]$ is thus unlikely due to geometrical restrictions in these AeTt compounds. For smaller hydrogen contents, however, the electronic flexibility of the $\pi^{*}$ electron reservoir seems sufficient and only some strengthening of existing Si-Si and $\mathrm{Ge}-\mathrm{Ge}$ bonds are found instead of forming new ones. Yet another solution to the optimization of space requirements and electronic structure is found in $\mathrm{NdGaH}_{1+\mathrm{x}}$, where hydrogen bridges two gallium zigzag chains approximately halfway between them ${ }^{[19]}$

To conclude this geometric reasoning, the combination of large alkaline earth and small tetrel atoms favors high hydrogen contents and single polyanionic chains, e. g. ${ }_{\infty}^{1}\left[\mathrm{SiH}^{-}\right]$in $\mathrm{BaSiH}_{2-x},{ }^{[5]}$ while small alkaline earth and large tetrel atoms should support the maximum degree of polyanion condensation. However, the tendency of the tetrel atoms to form stable covalent bonds to hydrogen limits this endeavor as seen for the inertness towards hydrogen (SrSn) or tendency to decomposition $(\mathrm{SrPb}, \mathrm{BaPb}){ }^{[5]}$ Varying the combinations of alkaline earth and tetrel atoms or substituting them by other elements gives ample opportunity to synthesize tetrel-tetrel and tetrel-hydrogen bonds in the solid. Such considerations may serve as a guide for the search for further compounds with new main group element-hydrogen entities or higher hydrogen content.

\section{Acknowledgements}

We thank Dr. Patrick Wenderoth for the synthesis of $\mathrm{SrSi}$ samples, Dr. Thomas Hansen for help with neutron diffraction, Dr. Gerald Wagner for help with practical TEM work, the Institute Laue-Langevin and Helmholtz Zentrum Berlin for providing beamtime and the Deutsche Forschungsgemeinschaft (Grant Ko1803/8-1) and the Fonds der Chemischen Industrie (Grant 194371) for financial support.

Keywords: Zintl phase $\cdot$ metal hydride $\cdot$ neutron diffraction $•$ electron diffraction $\cdot$ hydrogen

[1] U. Häussermann, V. F. Kranak, K. Puhakainen, Struct. Bond. 2011, 139, 143-161.

[2] H. Fahlquist, D. Noreus, S. Callear, W. David, I. F. William, B. C. Hauback, J. Am. Chem. Soc. 2011, 133, 14574-14577.

[3] J.-N. Chotard, W. S. Tang, P. Raybaud, R. Janot, Chem. - Eur. J. 2011, 17, 1521-3765.

[4] W. S. Tang, J.-N. Chotard, P. Raybaud, R. Janot, J. Phys. Chem. C 2014, 118, 3409-3419. 
[5] H. Auer, R. Guehne, M. Bertmer, S. Weber, P. Wenderoth, T. C. Hansen J. Haase, H. Kohlmann, Inorg. Chem. 2017, 56, 1061-1071.

[6] N. Ohba, M. Aoki, T. Noritake, K. Miwa, S.-i. Towata, Phys. Rev. B 2005, 72, 075104.

[7] P. Royen, R. Schwarz, Z. Anorg. Allg. Chem. 1933, 211, 412-422.

[8] V. F. Kranak, M. J. Evans, L. L. Daemen, T. Proffen, M. H. Lee, O. F. Sankey, U. Häussermann, Solid State Sci. 2009, 11, 1847-1853.

[9] Further details on the crystal structure investigations may be obtained from the Fachinformationszentrum Karlsruhe, 76344 EggensteinLeopoldshafen, Germany (fax: (+49)7247-808-666; e-mail: crysdata@fiz-karlsruhe.de), on quoting the depository number numbers CSD-433047, and -433048.

[10] M. Armbruster, M. Wörle, F. Krumeich, Z. Anorg. Allg. Chem. 2009, 635 1758-1766.

[11] D. Schiferl, C. S. Barrett, J. Appl. Crystallogr. 1969, 2, 30-36.

[12] A. Brown, S. Rundqvist, Acta Crystallogr. 1965, 19, 684-685.

[13] I. J. Maley, D. H. Brown, R. M. Ibberson, C. R. Pulham, Acta Crystallogr., Sect. B: Struct. Sci. 2008, 64, 312-317.

[14] V. P. Ting, P. F. Henry, H. Kohlmann, C. C. Wilson, M. T. Weller, Phys. Chem. Chem. Phys. 2010, 12, 2083-2088.

[15] E. C. Reyes, E. D. Stalder, C. Mensing, S. Budnyk, R. Nesper, J. Phys. Chem. C 2011, 115, 1090-1095.

[16] E. C. Reyes, R. Nesper, J. Phys. Chem. C 2012, 116, 2536-2542.

[17] I. M. Kurylyshyn, T. F. Fässler, A. Fischer, C. Hauf, G. Eickerling, M. Presnitz, W. Scherer, Angew. Chem. Int. Ed. 2014, 53, 3029-3032.

[18] H. Auer, D. Wallacher, T. C. Hansen, H. Kohlmann, Inorg. Chem. 2017, 56, 1072-1079.

[19] J. Ångström, R. Johansson, T. Sarkar, M. H. Sorby, C. Zlotea, M. S. Andersson, P. Nordblad. R. H. Scheicher, U. Häussermann, M. Sahlberg, Inorg. Chem. 2016, 55, 345-352 
Entry for the Table of Contents (Please choose one layout)

Layout 1:

\section{COMMUNICATION}

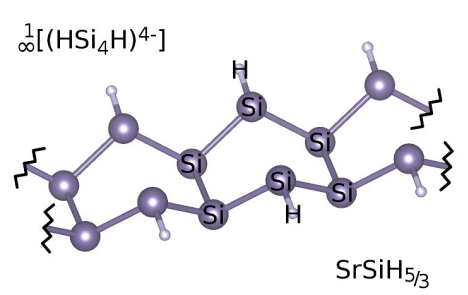

The Zintl phases SrSi, BaSi and BaGe form hydrides $A e T t \mathrm{H}_{5 / 3-\mathrm{x}}, \mathrm{Ae}=\mathrm{Sr}$, Ba; $\mathrm{Tt}=\mathrm{Si}, \mathrm{Ge}$, with new single- and double-chain polyanions characterized by additional tetrel-hydrogen and tetrel-tetrel bonds.
Henry Auer, Robert Schlegel, Oliver Oeckler, and Holger Kohlmann*

Page No. - Page No.

Structural and Electronic Flexibility in Hydrides of Zintl Phases from TetrelHydrogen to Tetrel-Tetrel Bonds

Layout 2:

\section{COMMUNICATION}

((Insert TOC Graphic here))
Author(s), Corresponding Author(s)*

Page No. - Page No.

Title

Text for Table of Contents 